\title{
Duloxetine in the management of diabetic peripheral neuropathic pain
}

\author{
This article was published in the following Dove Press journal: \\ Patient Preference and Adherence \\ I8 July 20 I I \\ Number of times this article has been viewed
}

\author{
Michelle J Ormseth \\ Beth A Scholz \\ Chad S Boomershine \\ Division of Rheumatology and \\ Immunology, Vanderbilt University, \\ Nashville, TN, USA
}

Correspondence: Chad S Boomershine Division of Rheumatology and Immunology, Vanderbilt University, T3219 MCN, II6I 2 Ist Ave South, Nashville, TN 37232, USA

Tel +l 6153224746

Fax + I 6153226248

Email chad.boomershine@vanderbilt.edu

\begin{abstract}
Diabetic neuropathy affects up to $70 \%$ of diabetics, and diabetic peripheral neuropathic pain (DPNP) is the most common and debilitating of the diabetic neuropathies. DPNP significantly reduces quality of life and increases management costs in affected patients. Despite the impact of DPNP, management is poor with one-quarter of patients receiving no treatment and many treated with medications having little or no efficacy in managing DPNP. Duloxetine is one of two drugs approved by the United States Food and Drug Administration for DPNP management. Duloxetine is a serotonin and norepinephrine reuptake inhibitor (SNRI) proven safe, effective, and cost-saving in reducing DPNP symptoms at a dose of $60 \mathrm{mg} /$ day. Duloxetine doses greater than $60 \mathrm{mg}$ /day for DPNP management are not recommended since they are no more efficacious and associated with more side effects; addition of pregabalin or gabapentin for these patients may be beneficial. Side effects of duloxetine are generally mild and typical for the SNRI class including nausea, dizziness, somnolence, fatigue, sweating, dry mouth, constipation, and diarrhea. Given its other indications, duloxetine is a particularly good choice for DPNP treatment in patients with coexisting depression, anxiety, fibromyalgia, or chronic musculoskeletal pain. Duloxetine treatment had no clinically significant effect on glycemic control and did not increase the risk of cardiovascular events in diabetes patients. However, duloxetine use should be avoided in patients with hepatic disease or severe renal impairment. Given its safety, efficacy, and tolerability, duloxetine is an excellent choice for DPNP treatment in many patients.
\end{abstract}

Keywords: duloxetine, diabetic peripheral neuropathic pain, review, treatment

\section{Introduction}

Diabetes is a common and costly disease in the US. ${ }^{1}$ In 2007 , it was estimated that 23.6 million US residents had diabetes. ${ }^{1}$ The prevalence of diabetes is particularly high in the fastest growing segment of the US population, individuals 60 years of age or older, with estimates of $20.9 \%$ in this group. ${ }^{2}$ Neuropathy is a common consequence of diabetes, affecting $60 \%-70 \%$ of diabetics. ${ }^{1}$ Diabetic neuropathies are a family of nerve disorders classified as peripheral, autonomic, proximal, and focal. ${ }^{3}$ Peripheral neuropathy is the most common diabetic neuropathy, estimated to occur in $47 \%$ of diabetics when nerve conduction testing is used for diagnosis. ${ }^{4}$ Peripheral neuropathies manifest with painful or painless symptoms, and many diabetic patients experience both. Neuropathic pain is defined by the International Association for the Study of Pain as "Pain arising as a direct consequence of a lesion or disease affecting the somatosensory system." ${ }^{5}$ Peripheral neuropathic pain occurs when a lesion or dysfunction affects the peripheral nervous system. ${ }^{6}$ The epidemiology of diabetic peripheral neuropathic 
pain (DPNP) has not been extensively studied, as epidemiologic studies have historically not differentiated between peripheral neuropathy patients with and without pain. However, DPNP is estimated to occur in $25 \%$ of all diabetics, ${ }^{6}$ and up to $50 \%$ of those with diabetic neuropathies. ${ }^{7}$ DPNP is particularly problematic in diabetic patients older than 60 years of age, with over half experiencing constant, daily pain that can substantially interfere with functional status. ${ }^{8}$

DPNP presents a unique challenge in patient management and should be considered a clinically distinct syndrome from diabetic peripheral neuropathy. ${ }^{9}$ DPNP can have debilitating consequences with a significant impact on patient quality of life (QOL) and diabetes management costs. ${ }^{7,10}$ The total annual direct cost of treating DPNP in the US has been estimated at US\$237 million (2001) or US\$306.08 per patient. ${ }^{9}$ However, the total direct and indirect DPNP-related costs to patients and society are much higher, estimated to be US\$3000-\$4000 (2009) per patient per year. ${ }^{11}$ In addition to monetary costs, DPNP can complicate diabetes treatment. DPNP often limits a patient's ability to exercise or walk, both of which have been shown to improve glucose management. ${ }^{12,13}$ DPNP often significantly interferes with sleep, ${ }^{14}$ and lack of sleep has been shown to have a negative impact on glycemic control. ${ }^{15}$ Due to its impact on patient health, the American Diabetes Association recommends screening for diabetic peripheral neuropathy in all patients at diagnosis and annually thereafter. ${ }^{13}$

Despite the impact and prevalence of DPNP, it remains undertreated and poorly managed. A 2007 survey showed that nearly $50 \%$ of diagnosed diabetic patients had not discussed DPNP or its symptoms with their clinician, ${ }^{16}$ and a 2004 study found that almost one-quarter of patients with DPNP had received no treatment for their pain. ${ }^{17}$ The DPNPassociated pain of even those patients whose glucose levels are well managed often goes untreated. ${ }^{18}$ Clinicians cannot assume that diabetic patients with good glycemic control will not have DPNP, as a recent study found that $17 \%$ of patients with DPNP had $\mathrm{HbA}_{1 \mathrm{c}}$ levels $<7 \%$, ${ }^{19}$ and painful diabetic neuropathy may occur just after strict glycemic control. ${ }^{20,21}$ Clinicians who do treat DPNP in their patients often use agents that are ineffective for managing neuropathic pain. A study of 55,686 patients with painful peripheral neuropathies found that the majority received short-acting opioids for treatment (53.2\%). ${ }^{17}$ The next largest percentage (39.7\%) were being treated with nonsteroidal anti-inflammatory drugs (NSAIDs) which have no effect on neuropathic pain and are a significant source of renal, gastrointestinal, and cardiovascular toxicity in diabetic patients. Two other drug classes with little or no efficacy in managing neuropathic pain, benzodiazepines and selective serotonin reuptake inhibitors (SSRIs), were also used to treat many patients $(21.1 \%$ and $14.2 \%$ of patients, respectively). The two classes of medications with the best evidence for efficacy in treating neuropathic pain, anticonvulsants and tricyclic antidepressants (TCAs), were used by the smallest percentage of patients (11.1\% and $11.3 \%$, respectively; this study predated the widespread use and availability of serotonin and norepinephrine reuptake inhibitors [SNRIs]).

\section{DPNP treatment options}

The first and most important treatment for all DPNP patients is maintaining glucose concentrations within the normal range. Tight glycemic control can prevent progression of diabetic neuropathy, ${ }^{22,23}$ and multiple studies have shown that improving glycemic control can reduce pain in DPNP patients. ${ }^{24-26}$ However, DPNP commonly occurs even in patients with good glycemic control, ${ }^{18}$ and pharmacologic treatments directed at pain are often necessary to manage DPNP. Two medications are currently approved by the US Food and Drug Administration (FDA) for the management of DPNP; duloxetine and pregabalin. Duloxetine is an SNRI indicated for the treatment of multiple diseases including major depressive disorder, generalized anxiety disorder, fibromyalgia, and chronic musculoskeletal pain. ${ }^{27}$ Duloxetine is thought to reduce the perception of pain by increasing the activity of descending pain pathways that dampen pain signals arising from the periphery and being relayed through the spinal cord dorsal horn. ${ }^{28}$ Given its other indications, duloxetine may be a good choice for DPNP patients with co-existing mood disorders and/or chronic musculoskeletal pain. Pregabalin is an anticonvulsant thought to reduce pain by binding to alpha2-delta subunits of presynaptic neuronal calcium channels and reducing the release of excitatory neurotransmitters involved in pain perception. In addition to DPNP, pregabalin is indicated for the management of post-herpetic neuralgia, fibromyalgia, and as an adjunct therapy for epilepsy. ${ }^{29}$

Duloxetine and pregabalin were both recommended firstline medications for managing DPNP in consensus treatment guidelines. ${ }^{30}$ While not FDA indicated, TCAs and the longacting opioid oxycodone CR were also listed as first-tier medications in these guidelines based on the results of at least two randomized control trials (RCTs) in DPNP. However, given the risks associated with opioids we recommend great caution in their use particularly in older patients. Second-tier agents with only a single DPN RCT to recommend them included the SNRI venlafaxine ER, the older 
alpha 2-delta antagonist gabapentin, anticonvulsants carbamazepine and lamotrigine, and tramadol, a weak opioid agonist with SNRI-activity. Other recommended treatments included the anticonvulsants phenytoin and topiramate, the antidepressants bupropion, citalopram, and paroxetine, and topical agents including capsaicin and lidocaine. Separate, newly published, DPNP treatment guidelines list pregabalin as the only recommended first-tier treatment choice, with duloxetine, venlafaxine, amitriptyline, gabapentin, valproate, opioids (morphine sulfate, tramadol, and oxycodone controlled-release), and capsaicin all considered second-tier options likely to be effective. ${ }^{31} \mathrm{~A}$ third-tier recommendation for combination therapy with gabapentin and venlafaxine was based on efficacy seen in a group of DPNP patients who had previously failed gabapentin monotherapy. ${ }^{32}$ Given the differences in mechanism of action, combined use of duloxetine and pregabalin may be a reasonable alternative in DPNP patients with an inadequate response to pregabalin, but RCTs are needed to determine the risks and benefits of combination therapy with these agents.

\section{Efficacy of duloxetine in the treatment of DPNP Phase III placebo controlled trials}

Three Phase III RCTs have demonstrated the efficacy of duloxetine in the management of DPNP in adults (no studies have evaluated children) (Table 1). ${ }^{33-35}$ The first trial enrolled 348 patients from 26 centers internationally. ${ }^{33}$ Inclusion criteria included patients with bilateral peripheral neuropathic pain caused by type 1 or type 2 diabetes mellitus who had mean scores of $\geq 4$ when assessed for 24-hour average pain severity on an 11-point Likert scale and stable glycemic control. The Michigan Neuropathy Screening Instrument (MNSI) was used to confirm the diagnosis of diabetic peripheral neuropathy. ${ }^{36}$ Exclusion criteria for this study included comorbid psychiatric illness (specifically major depressive disorder, dysthymic disorder, generalized anxiety disorder, alcohol or eating disorders, mania, bipolar, disorder, or psychosis as defined by Diagnostic and Statistical Manual of Mental Disorders [DSM] IV criteria utilizing the Mini International Neuropsychiatric Interview [MINI]), the presence of comorbid conditions that could also cause pain (such as phantom limb pain or peripheral vascular disease), endstage renal disease (either prior transplant or ongoing dialysis), substance abuse, or use of prescription opioids, fluoxetine, or monoamine oxidase inhibitors (MAOIs). The primary endpoint was change in weekly mean 24-hour Average Pain Scores extrapolated from daily patient diaries, with a 30\% improvement from baseline considered a therapeutic response. Responses based on 50\% reduction from baseline were also reported. Secondary endpoints included change in scores on: mean weekly worst and nighttime pain from daily patient diaries, Brief Pain Inventory, ${ }^{37}$ Short Form McGill Pain Questionnaire (a scale measuring various characteristics of pain ranging from 0 [none] to 3 [severe]), ${ }^{38}$ Dynamic Mechanical (Brush) Allodynia (measured on a scale of 0 [no pain] to 3 [severe pain] after brush stroke administered by study personnel at a standardized anatomic location), ${ }^{39}$ and Hamilton Depression Rating Scale (17 multiple-choice item questionnaire). ${ }^{40}$ The Brief Pain Inventory (BPI) is a 32-question survey used for patients to report pain quantitatively through visual analog scales as well as qualitatively through questions pertaining to impairment with usual daily activities due to pain. ${ }^{37}$ Further secondary outcomes included scores on the Clinical Global Impression of Severity Scale (CGI, a scale measuring the clinician's impression of change in disease severity ranging from 1 [normal, not at all ill] to 7 [among the most extremely ill patients]) and Patient Global Impression of Improvement (PGI-I, a scale measuring patient response to therapy ranging from 1 [very much better] to 7 [very much worse]). ${ }^{41}$

Study treatment groups included placebo, duloxetine $60 \mathrm{mg}$ once daily ( $\mathrm{mg} /$ day), or duloxetine $60 \mathrm{mg}$ twice daily. The study began with a 3-day dose-escalation phase during which all duloxetine-assigned patients took $60 \mathrm{mg} /$ day, followed by an increase to $60 \mathrm{mg}$ twice daily in the $60 \mathrm{mg}$ twice daily group. The average age of enrolled patients was 58.8 years. Mean duration of diabetes in the cohort was 13.8 years, and $99.7 \%$ were Caucasian. Demographic data was comparable between the study groups with the exception that the placebo group had a slightly higher baseline MNSI score (5.2 vs 4.8 and 5.0 in other groups). While this baseline difference was statistically significant $(P=0.036)$, the difference is not clinically significant since any score $>2.5$ is considered indicative of diabetic peripheral neuropathy.

Both duloxetine treatment groups met the primary trial endpoint with significantly greater percentages of patients having $\geq 30 \%$ reductions in 24 -hour average pain severity compared with the placebo treatment group $(68.14 \%$ for duloxetine $60 \mathrm{mg} /$ day, $64.04 \%$ for duloxetine $60 \mathrm{mg}$ twice daily and $43.36 \%$ for placebo; $P<0.001$ and $P=0.002$, respectively, for $60 \mathrm{mg} /$ day and $60 \mathrm{mg}$ twice daily groups vs placebo) (Table 1). A $\geq 50 \%$ reduction in 24-hour average pain was seen in $50 \%$ of patients treated with duloxetine $60 \mathrm{mg} /$ day, $39 \%$ of patients treated with duloxetine $60 \mathrm{mg}$ twice daily and $30 \%$ of patients treated with placebo 
Table I Percentage of patients with reduced 24-hour average pain severity in duloxetine DPNP trials

\begin{tabular}{|c|c|c|c|c|c|c|}
\hline \multirow{3}{*}{$\begin{array}{l}\text { Percent pain } \\
\text { reduction }\end{array}$} & \multirow[t]{3}{*}{ Trial } & \multicolumn{4}{|c|}{ Treatment } & \multirow{3}{*}{$\begin{array}{l}\text { All duloxetine groups significantly } \\
\text { different from placebo }\end{array}$} \\
\hline & & \multicolumn{3}{|c|}{ Duloxetine daily dose } & \multirow[t]{2}{*}{ Placebo } & \\
\hline & & $20 \mathrm{mg}$ & $60 \mathrm{mg}$ & $120 \mathrm{mg}$ & & \\
\hline \multirow[t]{2}{*}{$\geq 30 \%$} & Raskin et $\mathrm{al}^{33}$ & & $68.14 \%$ & $64.04 \%$ & $43.36 \%$ & Yes \\
\hline & Wernicke et $\mathrm{al}^{35}$ & & $63 \%$ & $69 \%$ & $42 \%$ & Yes \\
\hline \multirow[t]{3}{*}{$\geq 50 \%$} & Raskin et $\mathrm{al}^{33}$ & & $50 \%$ & $39 \%$ & $30 \%$ & Unreported \\
\hline & Goldstein et $\mathrm{al}^{34}$ & $41 \%$ & $49 \%$ & $52 \%$ & $26 \%$ & Yes \\
\hline & Wernicke et $\mathrm{al}^{35}$ & & $43 \%$ & $53 \%$ & $27 \%$ & Yes \\
\hline
\end{tabular}

Abbreviation: DPNP, diabetic peripheral neuropathic pain.

(Table 1). Pain improvements were achieved by the end of week 1 and sustained throughout the 12-week study period in duloxetine-treated patients. Secondary endpoints including BPI, ${ }^{37} \mathrm{CGI},{ }^{41}$ and PGI-I ${ }^{41}$ scores also demonstrated statistically significant improvement in both duloxetine treatment groups compared with placebo. While changes in dynamic mechanical allodynia scores did not achieve statistical significance, this measure has only been validated for reproducibility by a single administrator and it is likely that performance differences between study sites resulted in high variability that limited the ability to observe treatment differences. ${ }^{42}$ Also, given the dynamic allodynia rating at baseline of less than 1 on a scale of $0-3$, the patients did not have much room for improvement on this measure (mean change was -0.14 for placebo and duloxetine $60 \mathrm{mg}$ twice daily groups and -0.22 for the duloxetine $60 \mathrm{mg}$ daily group). Path analysis was utilized to separate the direct analgesic effect of duloxetine $60 \mathrm{mg} /$ day from pain improvement due to improvement in mood. This analysis concluded that over $90 \%$ of the analgesic effect of duloxetine at either dose was direct compared with placebo (98\% for $60 \mathrm{mg}$ twice daily and $92.7 \%$ for $60 \mathrm{mg} /$ day) and not attributable to improvement in mood. This result is not unexpected since patients with comorbid psychiatric illness were excluded from the study, and it is unlikely that patients had mood symptoms at baseline.

The second published duloxetine DPNP trial was 12-weeks in duration and enrolled 457 patients. ${ }^{34}$ This trial studied male and female patients with DPNP due to either type 1 or type 2 diabetes mellitus to compare the effect of duloxetine at doses of $20 \mathrm{mg} /$ day, $60 \mathrm{mg} /$ day, and $120 \mathrm{mg} /$ day (60 mg twice daily) to placebo. Subjects were enrolled through multiple sites within the US, and inclusion and exclusion criteria were similar to the first study. The average age of participants in all treatment groups was approximately 60 years, and, while genders were fairly evenly distributed, men outnumbered women in some treatment groups. The primary endpoint of the trial was change in mean weekly 24-hour average pain scores from baseline to endpoint based on data extrapolation from patient symptom diaries. While $\geq 30 \%$ reductions in average pain scores were not reported, $\geq 50 \%$ reductions for all groups were given. Secondary endpoints also based on symptom diary entries were change in mean weekly average daily and nighttime pain severity, and worst pain severity. BPI (severity and interference portions), ${ }^{37}$ CGI,,${ }^{41}$ PGI-I, ${ }^{41}$ SF-MPQ,${ }^{38}$ Dynamic Allodynia, ${ }^{36}$ Short Form Health Status Survey (SF-36, contains both physical and mental components to assess global patient function), ${ }^{43}$ and the EuroQol Group EQ-5D Quality of Life scale (measures impact on mood, pain, and impact on ability to perform various activities of daily living) ${ }^{44}$ were other secondary endpoints. Change in mood symptoms was monitored via the Beck Depression Inventory-II (BDI) ${ }^{45}$ and the Beck Anxiety Inventory (BAI). ${ }^{46}$

A linear, dose-dependent effect of duloxetine in reducing pain was seen consistently during the 12-week study period. The primary study endpoint of reduction in mean weekly 24-hour average pain score from baseline was reached in duloxetine 60 and $120 \mathrm{mg} /$ day groups, but efficacy of $20 \mathrm{mg} /$ day compared with placebo did not reach statistical significance and $120 \mathrm{mg}$ /day was not significantly better than $60 \mathrm{mg} /$ day. However, the percentage of patients with $a \geq 50 \%$ pain reduction in 24 hour average pain scores was significantly higher in all duloxetine treatment groups compared with placebo (41\% in the duloxetine $20 \mathrm{mg}$ /day group, $49 \%$ in the $60 \mathrm{mg} /$ day group, and $52 \%$ in the $120 \mathrm{mg} /$ day group vs $26 \%$ in the placebo group, $P<0.05$ for all activetreatment groups vs placebo) (Table 1). Change in BDI and BAI scores were used to estimate pain reduction due to duloxetine-mediated improvement in depression and anxiety symptoms, respectively. The direct analgesic effect was estimated to be $94.8 \%$ versus an indirect effect of $0.2 \%$ due to improvement in depression and $5.1 \%$ due to anxiety improvement in patients treated with duloxetine $60 \mathrm{mg} /$ day versus placebo $(P=0.003)$. The direct analgesic treatment effect in this trial was similar to the $92.7 \%$ direct effect seen 
for the $60 \mathrm{mg} /$ day duloxetine treatment group in the first treatment trial. ${ }^{33}$

The third Phase III RCT to be published investigating duloxetine for the management of DPNP compared duloxetine at doses of 60 and $120 \mathrm{mg} /$ day (60 mg twice daily) to placebo over 12 weeks. ${ }^{35}$ This study enrolled 334 patients from 28 international study centers. Inclusion and exclusion criteria were very similar to the previous two duloxetine studies, with patients required to have typical DPNP pain (symmetric and starting in feet) daily for at least 6 months, MSNI scores of $\geq 3$, a mean baseline score of $\geq 4$ when assessed by 24-hour average pain severity on the 11-point Likert scale from the patient diary, and stable glycemic control. Patients with comorbid psychiatric disease based on DSM-IV criteria were excluded as were patients taking medications known to cause neuropathy, those with substance abuse disorders, or those using MAOIs or fluoxetine. As in the previously discussed trials, use of only certain additional analgesic medications during the trial were allowed, limited to aspirin up to $325 \mathrm{mg} /$ day and acetaminophen up to $4 \mathrm{~g} /$ day. Randomization occurred after two initial study visits, during which baseline data was gathered. The primary endpoint was reduction in weekly mean 24-hour average pain scores calculated using patient symptom diary entries. Protocol-specified response at endpoint was defined as a $\geq 30 \%$ reduction from baseline to endpoint in the 24 -hour average pain score. Rates for $\geq 50 \%$ reductions in 24 -hour average pain response were also reported for all treatment groups. Secondary endpoints were identical to the aforementioned duloxetine studies. Pertinent demographics of the study population included $61.1 \%$ male, $78.1 \%$ Caucasian, and a mean age of 60.7 years.

Unlike the first published study, the baseline MNSI score was similar amongst all groups, but the BPI average pain interference score was slightly lower at baseline in the placebo group (4.2 vs 4.7 and 5.0 for the 60 and $120 \mathrm{mg}$ /day groups, respectively). This trial confirmed the findings of the previous two studies by showing that both duloxetine treatment doses met the primary and most secondary end points compared with placebo. Specifically, the pain score decreased by an average of $1.32 \mathrm{in}$ the duloxetine $60 \mathrm{mg} /$ day group and 1.44 in the $120 \mathrm{mg} /$ day group compared with placebo. $\mathrm{A} \geq 30 \%$ reduction in 24-hour average pain response was achieved by $63 \%$ in the duloxetine $60 \mathrm{mg}$ /day group and $69 \%$ in the duloxetine $60 \mathrm{mg}$ twice daily group versus $42 \%$ in the placebo-treatment group $(P=0.003$ and $P<0.001$ vs placebo, respectively). Significantly more patients in both duloxetine treatment groups also had $\geq 50 \%$ reductions in 24-hour average pain response compared with placebo (43\% for $60 \mathrm{mg} /$ day and $53 \%$ for $60 \mathrm{mg}$ twice daily vs $27 \%$ for placebo, $P<0.05$ and $P<0.001$, respectively). A $\geq 50 \%$ pain improvement is considered to be a "substantial" improvement in pain that is more significant to the day-to-day life of patients than a $\geq 30 \%$ improvement which is considered only a "moderately important" improvement that indicates a minimal clinically significant difference. ${ }^{47}$ This trial also identified a significant difference not seen in the previously discussed trials between the $60 \mathrm{mg} /$ day and $120 \mathrm{mg} /$ day duloxetine groups, with the higher dosing group using significantly less additional analgesic medications (ie, acetaminophen or aspirin). The two previously discussed Phase III trials reported significantly less additional analgesic use between the duloxetine and placebo treatment groups, but not between duloxetine dosage groups. ${ }^{33,34}$ Changes in measurements of dynamic allodynia were not statistically significant, but this is not surprising given the low average baseline scores ( 0.2 and 0.3 out of 3 for placebo and both duloxetine groups, respectively) and the previously discussed problems with inter-rater variability. ${ }^{42}$ The lack of improvement in HAMD17 scores was attributed to low baseline depression symptoms, probably a reflection of the exclusion of patients with comorbid major depressive disorder (MDD).

\section{Longer-term open-label extension studies}

The three previously described Phase III RCTs were each extended into open-label studies to monitor the efficacy of duloxetine in treating DPNP over longer time periods. ${ }^{48-50}$ In the first extension trial, 237 patients who completed the initial study period were re-randomized to receive either duloxetine $120 \mathrm{mg} /$ day (60 mg twice daily) or routine clinical care for 52 weeks. ${ }^{48}$ As in the original trial, there was a 3-day lead-in treatment period with duloxetine $60 \mathrm{mg} /$ day before escalation to $60 \mathrm{mg}$ twice daily in the duloxetine treatment group. Unlike in the RCTs, patients were permitted to reduce the duloxetine dose to $60 \mathrm{mg} /$ day if they experienced intolerable treatment emergent adverse events (TEAEs). Patients in the routine care group received medications to treat DPNP based on the preference of the investigators. The most commonly used treatments were gabapentin (57.9\%), amitriptyline (22.4\%), and venlafaxine (both immediate release and sustained release preparations were used, totaling $21.0 \%$ ). Unlike the RCTs, the primary endpoints were changes in SF-36 and EQ-5D scores to measure improvement in overall health and quality of life, respectively. The study found no significant difference in changes in outcome measure scores between patients receiving duloxetine and those receiving routing care. 
Unfortunately, no direct comparisons between duloxetine and specific routine-care drugs were made, likely due to the small number of patients in each routine-care drug group.

The second, longer-term, open-label extension study enrolled 337 patients after they had completed the initial 12-week duloxetine RCT study. ${ }^{49}$ As in the first trial, one group of patients was randomized to receive duloxetine $120 \mathrm{mg} /$ day (60 mg twice daily) while the other received routine clinical care for 52 weeks. However, in this trial routine care was not limited to specific medications but rather to "... therapies that the investigator and the patient believed would provide optimal benefit for the patient ..." Also, in this trial both treatment groups were permitted to use nonmedicinal therapies as desired. As in the previous open-label trial, changes in SF-36 and EQ-5D scores served as primary efficacy outcomes in the study. In contrast to the previous trial, data analysis showed a statistically significant improvement in the SF-36 bodily pain subscale score for the duloxetine treatment group versus the routine care group. However, no other differences were found for SF-36 global or subscale scores between the treatment groups. While both treatment groups experienced significant improvement in the EQ-5D score from baseline, patients in the routine-care group demonstrated significantly greater improvement compared with those treated with duloxetine. Unfortunately, no data were provided regarding specific treatments used in the routine treatment group or what doses of medications were employed to manage patients.

The third open-label extension study was similar in design to the second open-label extension trial, with 293 patients who had completed the initial study re-randomized to receive treatment over 52 weeks with duloxetine $120 \mathrm{mg} /$ day (with usual $60 \mathrm{mg} /$ day lead-in for 3 days) or routine care, with medications or other therapies used in the routine care group left to the discretion of the investigator. ${ }^{50}$ Change in SF-36 and EQ-5D scores were again used as primary study outcome measures, but this study was primarily meant to evaluate safety and long-term tolerability rather than efficacy. The efficacy results of this study will be discussed in this section, the safety and tolerability results are covered elsewhere in this review. In contrast to the preceding open-label extension studies, this trial found a statistically significant improvement in the SF-36 physical component summary score as well as SF-36 subscale scores for physical functioning, bodily pain, mental health, and vitality in patients treated with duloxetine compared with those receiving routine care. However, as in the other two open-label extension trials, no differences in EQ-5D scores were seen between the two treatment groups.
A different study group, still associated with Eli Lilly, evaluated the efficacy of duloxetine in treating DPNP over 26 weeks. ${ }^{51}$ The primary endpoint of the study was improvement in BPI average pain scores. This study was an openlabel design consisting of two stages. In the first stage, patients were placed on duloxetine $60 \mathrm{mg}$ /day (after a 1-week $30 \mathrm{mg} /$ day lead-in) for 7 weeks (weeks 1-8). In the second stage, patients who experienced at least a 30\% improvement in BPI average pain rating, termed responders, continued on this dose for an additional 26 weeks (weeks 8-34). Nonresponders (those with a $<30 \%$ improvement in pain) after the initial 8 weeks were moved to a "rescue" arm and had their duloxetine dose increased to $120 \mathrm{mg}$ /day as a single, once daily dose that was continued for the remaining 26 weeks. Patients in the $60 \mathrm{mg} /$ day group who lost their treatment response between weeks 7 and 12 could be moved to a separate "rescue arm" wherein their duloxetine dose was increased to $120 \mathrm{mg}$ /day during weeks 12 through 24 . A total of 184 patients completed the initial 8-week phase, and 53.2\% were identified as responders. Ultimately, 69 patients were assigned to the rescue arm. The improvement seen at 8 weeks (labeled "acute phase") was sustained throughout the 26-week period ("maintenance phase") with the exception of a transient increase in BPI in the responder group at the week 12 assessment. Of the responders, $66.7 \%$ had a $\geq 50 \%$ improvement in pain scores at the end of the trial. Of the nonresponders who transitioned to the rescue arm, $31.8 \%$ experienced $\mathrm{a} \geq 50 \%$ reduction in pain after their duloxetine dose was increased to $120 \mathrm{mg} /$ day. A number of secondary endpoints also indicated that duloxetine treatment significantly improved symptoms other than pain in responders, including global improvement, mood, walking ability, sleep, working ability, relations with other people, and enjoyment of life, and improvements made in the majority of these areas were maintained throughout the 34 weeks of the trial.

One other published study evaluated the safety of duloxetine in the treatment of DPNP. ${ }^{52}$ This trial was an open-label study that enrolled 449 patients from 36 international sites. In the study, patients were randomized to duloxetine $120 \mathrm{mg}$ /day dosed as either $120 \mathrm{mg}$ once daily or $60 \mathrm{mg}$ twice daily. Inclusion criteria were similar to the other studies, and all patients took duloxetine $60 \mathrm{mg}$ once daily for 1 week before dose escalation. Change in BPI and CGI-S scores were the primary efficacy endpoints, although the primary stated goal of the study was to evaluate safety and not efficacy. Both duloxetine treatment groups demonstrated significant improvement in BPI and CGI-S scores, but no significant betweengroup differences were seen for duloxetine dosing groups. 
The clinical relevance of the open-label studies that used a duloxetine dose of $120 \mathrm{mg} /$ day is questionable since the approved DPNP treatment dose for duloxetine is $60 \mathrm{mg}$ once daily and no compelling data suggest increased efficacy for treating DPNP at duloxetine doses higher than $60 \mathrm{mg} /$ day. However, the data derived from group means provide the basis for this assumption, and our experience with individual patients indicates that some DPNP patients may have additional clinical benefit with duloxetine doses above $60 \mathrm{mg}$ /day. While we do not recommend routine use of duloxetine doses above $60 \mathrm{mg} /$ day, higher doses may be beneficial to some patients. If clinicians chose to use higher than indicated duloxetine doses, we recommend possible adverse events be reviewed with patients and that close monitoring for adverse events be performed at regular intervals. It is also important to note that all the efficacy studies were conducted in association with Eli Lilly, the manufacturer of duloxetine. The similar study design used in all trials, likely due to the Lilly association, is beneficial since it makes direct comparisons between studies possible. However, this sponsorship could also be a liability since the affiliation may have influenced the interpretation of study data and thereby biased trial results. A recent Cochrane Review determined that 6 DPNP patients would need to be treated with $60 \mathrm{mg}$ /day of duloxetine for one patient to have a $50 \%$ reduction in pain after 12 weeks of therapy. ${ }^{53}$ It is interesting that this number needed to treat (NNT) of 6 for duloxetine treatment of DPNP is similar to the NNT of 8 that has been shown for duloxetine at $60 \mathrm{mg}$ /day for the management of fibromyalgia pain. ${ }^{53}$

\section{Safety of duloxetine in DPNP treatment}

\section{Treatment-emergent and serious adverse events seen in Phase III trials}

Table 2 shows the TEAEs and serious adverse events (SAEs) that were associated with duloxetine treatment in the three Phase III DPNP RCTs. ${ }^{33-35}$ The most common TEAEs were nausea, dizziness, somnolence, fatigue, sweating, dry mouth, constipation, and diarrhea. There was a dose-related increase in the discontinuation rate due to adverse events (AEs) seen across all three duloxetine DPNP studies. One study noted that the most common reason for discontinuation was nausea, and that $86 \%$ of patients who discontinued due to AEs did so during the first 4 weeks of the study. ${ }^{33}$ There was no significant difference in the percentage of patients who experienced SAEs between treatment groups. For the listed SAEs, no concerning safety signal was detected. However, specific concerns about cardiovascular $(\mathrm{CV})$ risk will be addressed in the next section.

\section{Cardiovascular disease-related adverse events}

There is particular concern for possible CV SAEs in diabetic patients treated with duloxetine. Diabetes mellitus has long been recognized as an independent risk factor for several forms of $\mathrm{CV}$ disease including coronary heart disease, stroke, peripheral arterial disease, cardiomyopathy, and congestive heart failure. ${ }^{54}$ Indeed, CV complications are now the leading causes of illness and death in the diabetic patient. ${ }^{55}$ Patients with diabetes are also predisposed to hypertension, ${ }^{56}$ with hypertension prevalence estimates ranging from $40 \%$ to $80 \%$ in patients with type 1 or type 2 diabetes, respectively. ${ }^{57,58}$ Hypertension is a known risk factor for $\mathrm{CV}$ disease, and duloxetine has been associated with slight increases in mean heart rate and blood pressure likely due to its inhibition of norepinephrine reuptake. ${ }^{59}$ Duloxetine could also potentially increase the risk of CV-related AEs due to drug-drug interactions, since duloxetine is known to interact with multiple cytochrome P450 enzymes including CYP1A2 and CYP2D6. ${ }^{27}$

An Eli Lilly sponsored study pooled data from the three Phase III DPNP trials of duloxetine to determine if patients with known cardiovascular disease might be at increased risk for serious adverse events (SAEs). ${ }^{60}$ Of the 1024 patients analyzed, 762 (74.4\%) had pre-existing CV disease. As would be expected, since patients with $\mathrm{CV}$ disease are more likely to have SAEs, a greater proportion of patients with pre-existing CV disease experienced SAEs compared with patients without known $\mathrm{CV}$ disease (4.6\% vs $1.5 \%$, respectively). However, comparing patients with known $\mathrm{CV}$ disease to one another, duloxetine-treated patients did not have a higher SAE rate compared with those treated with placebo. In fact, the $\mathrm{SAE}$ rate among patients treated with duloxetine was actually numerically lower and just missed statistical significance $(3.5 \%$ vs $6.7 \%$, respectively, $P=0.06$ ). Looking specifically at CV-related SAEs, eleven events were recorded in patients with known $\mathrm{CV}$ disease. Seven were reported in the duloxetine-treated group (two cases of hypertension and one case each of atrial fibrillation, myocardial infarction, ventricular extrasystoles, congestive heart failure, and coronary artery stenosis), and four were reported in the placebotreated group (two cases of hypertension and one case each of congestive heart failure and cerebrovascular accident). By comparison, only one $\mathrm{CV}$ disease-related SAE occurred in patients without known cardiovascular disease (second 


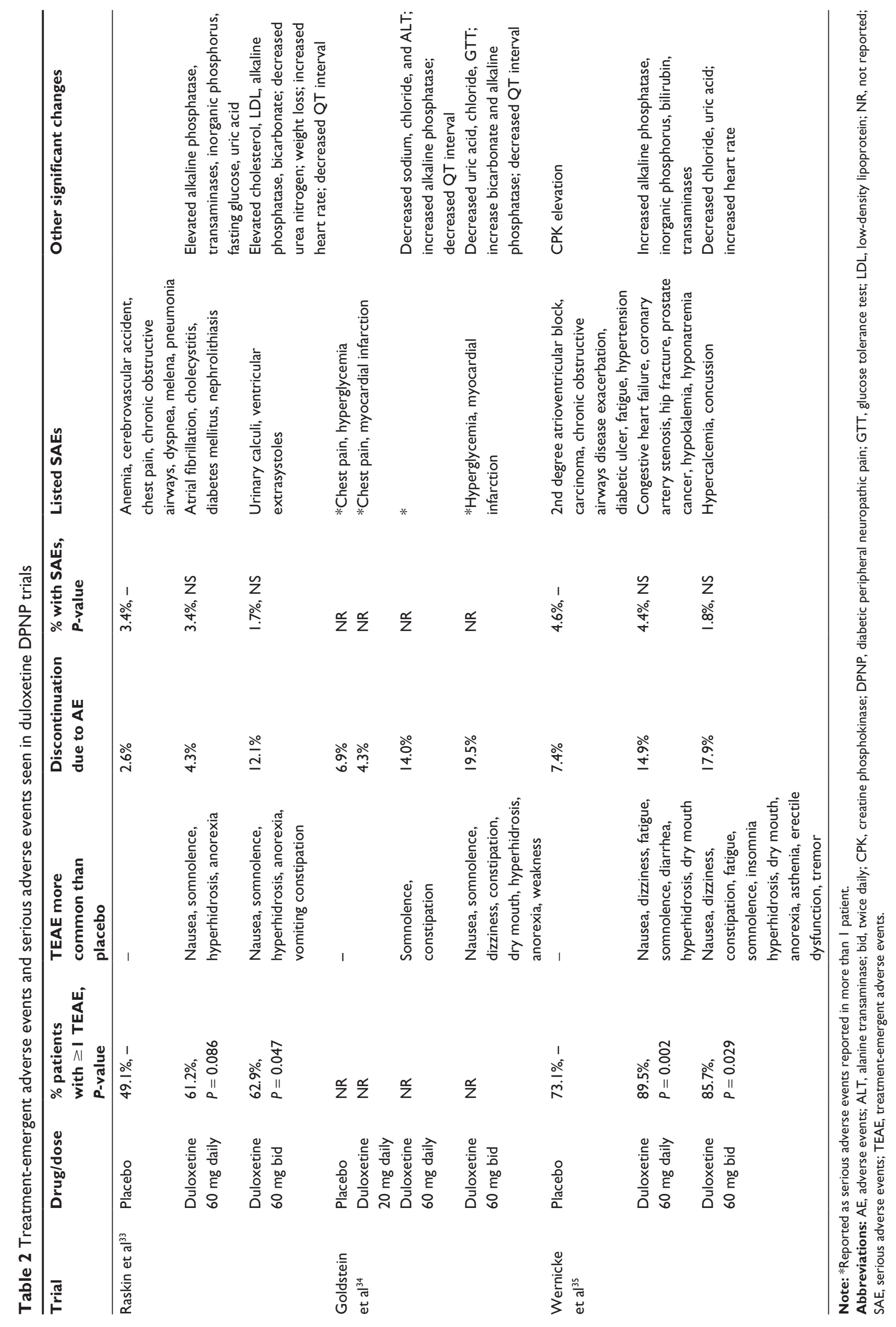


degree atrioventricular block), and this occurred in a placebo-treated patient. No significant differences were seen in rates of $\mathrm{CV}$ disease-related TEAEs in trial participants with $(8.4 \%$ in duloxetine and $9.9 \%$ in placebo) or without ( $8.6 \%$ in duloxetine and $5.7 \%$ in placebo) known preexisting $\mathrm{CV}$ disease, and no statistically significant differences in TEAE rates were seen between any groups $(P>0.1)$.

For patients on the approved duloxetine DPNP treatment dose of $60 \mathrm{mg}$ once daily, no statistically significant increase in mean heart rate was seen. Also, no significant change in systolic or diastolic blood pressure was seen in patients with known CV disease. Interestingly, a statistically significant increase in mean diastolic blood pressure was seen in duloxetine-treated patients without baseline $\mathrm{CV}$ disease compared with placebo-treated patients without $\mathrm{CV}$ disease (mean change: $-1.18 \mathrm{mmHg}$ for placebo treatment vs $+1.63 \mathrm{mmHg}$ for duloxetine treatment, $P<0.05$ ). However, it is important to note that patients with known hypertension entering these studies were required to have controlled blood pressure at baseline and this may have conferred protection against blood pressure increases to those with known cardiovascular disease. Also, the meta-analyses report average blood pressure changes and cannot be used to predict changes in blood pressure and heart rate that may occur in individual patients. For this reason, we recommend monitoring the blood pressure and heart rate of patients taking duloxetine periodically regardless of their history of $\mathrm{CV}$ disease.

Other antidepressants, primarily the TCAs, are known to increase CV TEAEs by increasing the QT interval and precipitating arrhythmias. However, there is no evidence that clinically significant increases in QT interval are associated with duloxetine use. On the contrary, two separate studies have shown small decreases in corrected QT interval with duloxetine treatment. ${ }^{33,34}$

\section{Worsening of glycemic control}

While selective serotonergic reuptake inhibitors (SSRIs) like fluoxetine can reduce hyperglycemia and increase insulin sensitivity, noradrenergic antidepressants like desipramine are known to promote hyperglycemia and insulin resistance. ${ }^{61}$ Thus, there is concern for worsening of glycemic control in the diabetic patient population taking a medication like duloxetine that inhibits norepinephrine reuptake. To determine whether duloxetine treatment is associated with worsening glycemic control in diabetics, a meta-analysis of the three Phase III duloxetine DPNP trials was performed..$^{62}$ This meta-analysis showed that during the initial 12-weeks of the trials patients treated with duloxetine on average had a modest, but non-significant, mean increase in fasting blood glucose compared with those treated with placebo $(+0.50 \mathrm{vs}$ $-0.11 \mathrm{mmol} / \mathrm{L}$, respectively, $P=0.064)$. Also, differences in changes in hemoglobin $\mathrm{A}_{1 \mathrm{C}}\left(\mathrm{HbA}_{1 \mathrm{C}}\right)$ levels were seen in duloxetine-treated patients compared to those treated with placebo. However, in the 52-week open label extension phase of these studies a statistically significant increase in mean fasting blood glucose ( +0.67 vs $-0.64 \mathrm{mmol} / \mathrm{L}, P<0.001)$ and $\mathrm{HbA}_{1 \mathrm{C}}(+0.52 \% \mathrm{vs}+0.19 \%, P<0.001)$ levels were seen in duloxetine-treated patients compared with those receiving routine care. ${ }^{62}$ While the average changes were small, larger changes could be seen in individual patients. For this reason, diabetes patients placed on duloxetine for management of their DPNP should be monitored for worsening of their glycemic control. However, from the available data it appears the risk of worsening glycemic with duloxetine treatment at the indicated DPNP treatment dose is small.

\section{General laboratory abnormalities}

Modest increases in liver function tests, bicarbonate and inorganic phosphorus associated with duloxetine treatment were seen in the three Phase III duloxetine DPNP trials but were not deemed by the authors to be clinically significant (Table 2). However, these figures compare mean changes and cannot be used to predict changes that may occur in individual patients. Hepatic failure, sometimes fatal, has been reported in patients treated with duloxetine. ${ }^{27}$ For this reason, patients should have baseline liver function testing to look for any liver disease. Duloxetine should not be used in patients with evidence of chronic liver disease or substantial alcohol use and we recommend periodic liver function testing be performed while patients are on duloxetine treatment. Since many patients with diabetes have renal disease that can affect electrolyte levels, and cases of hyponatremia have been reported with duloxetine use, ${ }^{27}$ we also recommend periodic monitoring of electrolytes while patients are treated with duloxetine particularly in the elderly who are at increased risk of electrolyte abnormalities.

One study found an increase in total and LDL cholesterol associated with duloxetine treatment, ${ }^{33}$ but this finding was not substantiated by the other two trials (Table 2). Regarding lipids, a meta-analysis of the three Phase III duloxetine DPNP trials showed that during the initial 12 weeks of the trials, duloxetine treatment was associated with a small decrease in serum triglycerides and small increases in high-density lipoprotein (HDL) cholesterol, low-density lipoprotein (LDL) cholesterol, and total cholesterol ${ }^{62}$ However, change in HDL cholesterol level was the only measure in 
duloxetine-treated patients that was statistically different from that seen in placebo-treated patients, and the difference was not clinically significant (+0.03 vs $0.00 \mathrm{mmol} / \mathrm{L}$, respectively, $P=0.008) .{ }^{62}$ Similarly, during the 52-week open-label extension phase of the DPNP duloxetine trials, there was a small numeric increase in serum levels of triglycerides, total and LDL cholesterol associated with duloxetine treatment, but only the change in total cholesterol levels was significantly different in duloxetine-treated patients compared with those who received routine care and once again the difference was not clinically significant $(+0.06 \mathrm{vs}-0.16 \mathrm{mmol} / \mathrm{L}$, respectively, $P=0.005) .{ }^{62}$ While HDL cholesterol levels slightly decreased in the duloxetine-treated group in the extension phase, levels in the routine care group dropped significantly more $(-0.01$ vs $-0.08 \mathrm{mmol} / \mathrm{L}$, respectively, $P=0.002) .{ }^{62}$ Taken together, there is no evidence that duloxetine treatment significantly impacts lipid levels in patients with DPNP. However, since diabetic patients are at increased risk for lipid abnormalities and $\mathrm{CV}$ events, evaluation and management of lipid abnormalities should be a part of routine care in these patients.

\section{Weight changes}

Weight loss was seen in one of the Phase III duloxetine DPNP studies. ${ }^{33}$ In this study, treatment with duloxetine at a dose of $60 \mathrm{mg}$ bid was associated with a mean weight loss of $0.9 \mathrm{~kg}$ compared with placebo $(P=0.006)$. No significant mean weight change was seen in patients treated with the approved duloxetine dose of $60 \mathrm{mg} /$ day. ${ }^{33}$ Pooled analysis of the three duloxetine DPNP treatment studies showed that duloxetine treatment was associated with an initial weight loss compared with placebo in the first 12 weeks of the trials (mean change $-1.03 \mathrm{vs}+0.03 \mathrm{~kg}$, respectively, $P<0.001) .{ }^{62}$ However, weight loss was not maintained in the 52-week extension study and, on average, patients in both duloxetine treatment and routine care groups gained weight $(+0.31$ and $+0.49 \mathrm{~kg}$, respectively, $P=0.531) .{ }^{62}$

\section{Fracture risk}

SSRIs have been linked to increased fracture risk due to their effects on bone metabolism. ${ }^{63}$ However, to our knowledge, increased fracture risk has not been linked to the use of SNRIs like duloxetine. There were no reports of fractures in the three Phase III duloxetine DPNP trials or open label extension studies. ${ }^{33-35,64-66}$ However, diabetic patients are known to be at increased risk for fractures compared with nondiabetics for a given bone mineral density, ${ }^{65}$ and all diabetics should be monitored for development of osteoporosis and aggressively managed if fragility fractures occur to prevent future fractures as part of routine care.

\section{Patient monitoring}

Baseline and periodic monitoring of blood pressure, pulse, electrolytes, renal, and liver function, and serum lipids are recommended while patients are being treated with duloxetine. Although no cases of suicidality were observed in the duloxetine DPNP trials, treatment with antidepressants is known to increase the risk of suicidality in some patients. ${ }^{66}$ Since suicidality is more likely to occur in patients with underlying psychiatric disorders, particularly bipolar disorder, it is recommended that patients with depressive symptoms be evaluated for bipolar disorder prior to initiating duloxetine and that patients treated with duloxetine be closely monitored for unusual changes in behavior or suicidality. ${ }^{27}$

\section{Contraindications}

According to the package insert, ${ }^{27}$ duloxetine should not be administered to patients with any hepatic insufficiency. Also, duloxetine is not recommended for patients with severe renal impairment (defined as an estimated creatinine clearance of $<30 \mathrm{~mL} / \mathrm{min}$ ). Finally, use should be avoided in patients with uncontrolled acute narrow angle glaucoma. Also, based on our clinical experience, we recommend that glaucoma patients be regularly monitored for changes in intraocular pressure while they are being treated with any selective serotonin reuptake inhibitor (SSRI) or SNRI.

\section{Drug interactions}

Concomitant use of MAOIs with all serotonergic drugs, including duloxetine, is contraindicated. Duloxetine should be used cautiously in combination with other serotonergic drugs, including TCAs, SSRIs, triptans, antipsychotics, or other dopamine antagonists, in order to decrease the risk of serotonin syndrome or neuroleptic malignant syndrome. ${ }^{27}$ Potent CYP1A2 and CYP2D6 inhibitors should also be avoided in combination with duloxetine as this may result in increased serum concentrations of duloxetine. Similarly, drugs with a narrow therapeutic index that are metabolized by the P450 CYP2D6 enzyme should be used with caution in combination with duloxetine since duloxetine may increase plasma concentration of these drugs. TCAs, phenothiazines, and type $1 \mathrm{C}$ antiarrhythmics should also be used with caution in combination with duloxetine. Thioridazine should not be used in combination with duloxetine. $^{27}$ 


\section{Patient acceptability}

There are several medications currently available with purported benefit in the treatment of DPNP, with duloxetine among the newer of these agents. ${ }^{30,31}$ While no published head-to-head trials comparing DPNP medications exist, multiple cost-effectiveness analyses have been performed on available data to determine the relative cost effectiveness of different DPNP medications. The first cost-utility comparison was formulated by an independent group of investigators at the University of Rochester in New York to determine the relative cost effectiveness of numerous medications used to treat DPNP. ${ }^{67}$ This analysis was undertaken without industry support or ties and therefore provides a rare third-party analysis that is likely to be objective. Making use of both published and unpublished data, a decision analytic model was used to compare desipramine $100 \mathrm{mg} /$ day, gabapentin $2400 \mathrm{mg} /$ day, pregabalin $300 \mathrm{mg}$ /day and duloxetine $60 \mathrm{mg} /$ day for the management of DPNP in terms of quality adjusted life years (QALY). Desipramine and duloxetine were found to be more efficacious and also less expensive compared with pregabalin and gabapentin. Specifically, duloxetine was calculated as having an incremental costeffectiveness ratio of US\$47,700 per QALY compared with desipramine, which was the reference medication against which the others were measured. While another costeffectiveness analysis of open-label extension studies showed a benefit for the use of duloxetine in DPNP treatment compared with other treatments, ${ }^{68}$ the results of this analysis should be interpreted cautiously since the analyzed studies used higher than indicated duloxetine doses $(120 \mathrm{mg} /$ day $)$ and the analysis was financed by Eli Lilly, the manufacturer of duloxetine.

A meta-analysis has also been used to indirectly compare the effectiveness of duloxetine, pregabalin, and gabapentin for the treatment of DPNP. ${ }^{69}$ These authors, who disclosed ties to Eli Lilly at the time of publication, identified no statistically significant difference in efficacy or tolerability between duloxetine and gabapentin. While duloxetine and pregabalin had similar efficacy in reducing 24 -hour pain scores, duloxetine was found to be superior to pregabalin with regards to TEAEs (specifically dizziness), whereas pregabalin was superior to duloxetine in improving global disease symptoms as assessed by PGI-I/C scores. The authors concluded that all three medications provided comparable efficacy and tolerability in DPNP patients and that selection among the three agents should be based on individual patient tolerance and preference. Based on its other indications and our clinical experience, we recommend duloxetine as a first line treatment for DPNP patients with coexisting depression or anxiety disorders and pregabalin or gabapentin as the first-line DPNP therapy in patients with concomitant sleep problems or seizure disorders.

Eli Lilly researchers investigating health care costs and adherence in patients taking duloxetine for DPNP using retrospective data found that a dose of $60 \mathrm{mg}$ /day was associated with maximal adherence and less health care costs over a 12-month study period compared with higher or lower duloxetine doses. ${ }^{70}$ However, the significance of this data is questionable since $60 \mathrm{mg} /$ day is the indicated dose of duloxetine for DPNP treatment, ${ }^{27}$ and all longerterm tolerability data for safety have been measured using $120 \mathrm{mg} /$ day of duloxetine. ${ }^{48-50}$ Other retrospective data has demonstrated that DPNP patients who use duloxetine continuously for 12 months had lower opioid use than either those who used duloxetine intermittently or who were treated with other typical medical therapies for DPNP (specifically, TCAs, venlafaxine, gabapentin, and pregabalin). ${ }^{71}$ Since opioids have significant TEAEs, such as somnolence and constipation, and a recent study found that older adults treated with opiates had increased risk for cardiovascular events, fracture risk, safety events requiring hospitalization, and risk of all-cause mortality ${ }^{72}$ minimizing opioid use is likely to be beneficial for overall patient health and well-being.

Cessation of duloxetine therapy can result in discontinuation-associated adverse events (DEAEs), typified by the discontinuation syndrome that is often seen when abruptly stopping drugs that inhibit serotonin reuptake. ${ }^{73}$ Eli Lilly researchers pooled data from patients after their participation in duloxetine treatment studies for DEAEs and found that DEAEs occurred in $44.3 \%$ of patients who abruptly stopped duloxetine. ${ }^{74}$ The most common DEAE was dizziness $(12.4 \%)$, followed by nausea $(5.9 \%)$, and headache $(5.3 \%)$. Other less commonly seen DEAEs included paresthesias, irritability, and nightmares. However, it is important to note that the majority of DEAEs occurred in patients treated with $120 \mathrm{mg} /$ day of duloxetine; the rates of DEAEs in patients taking $\leq 60 \mathrm{mg} /$ day of duloxetine were similar to those seen in patients treated with placebo. Also, the majority of DEAEs (65\%) resolved relatively quickly, within 7 days of duloxetine discontinuation. Finally, these DEAE rates were derived from duloxetine studies in patient with major depressive disorder and the applicability to DPNP patients is unknown. However, due to possible DEAE risk, a gradual dose reduction is recommended in patients who must discontinue duloxetine therapy regardless of the indication. ${ }^{27}$ 
A common issue that often arises in initiating duloxetine treatment is the best method to switch patients from an SSRI to duloxetine, specifically whether the SSRI should be gradually or abruptly discontinued. This issue was studied in a multicenter, open-label, randomized trial conducted in Europe. ${ }^{75}$ The study randomized 368 patients with major depressive disorder taking an SSRI to either begin a 2-week gradual discontinuation or abruptly stop their SSRI at the time duloxetine $60 \mathrm{mg} /$ day was started. The trial found no significant difference in the TEAE rates between gradual and abrupt discontinuation groups, and there were no significant differences in the adverse event discontinuation rate overall or the rate of study discontinuation due to any particular adverse event. Also, changes in vital signs, laboratory tests, and weight were not significantly different between the two discontinuation groups. Based on these data, there appears to be no need to taper an SSRI upon initiation of duloxetine treatment.

Other issues that commonly arise in initiating duloxetine treatment are the timing of administration and optimal starting dose. The duloxetine prescribing information gives no recommendation on the timing of duloxetine treatment. However, since nausea, dizziness, and somnolence were common TEAEs seen in DPNP trials, ${ }^{27}$ we typically recommend that patients take duloxetine with their evening meal or with a bedtime snack. The duloxetine prescribing information recommends starting with the indicated $60 \mathrm{mg}$ once daily dose. ${ }^{27}$ However, in our experience, many patients do not tolerate starting duloxetine at the indicated dose. The duloxetine prescribing information states that: "For patients for whom tolerability is a concern, a lower starting dose may be considered. Since diabetes is frequently complicated by renal disease, a lower starting dose and gradual increase in dose should be considered for patients with renal impairment." ${ }^{27}$ Based on the efficacy for reducing pain shown by duloxetine $20 \mathrm{mg}$ /day in DPNP patients, ${ }^{34}$ we typically start all patients on this dose. We recommend that patients are monitored for therapeutic response and tolerability at weekly or bi-weekly intervals, and that the dose is increased in 20-mg increments as needed and tolerated, to a maximum of $60 \mathrm{mg} /$ day. However, since the smallest dose Eli Lilly samples is $30 \mathrm{mg}$, we would start with $30 \mathrm{mg} /$ day if the patient requires samples.

\section{Conclusion and key points}

1. Duloxetine at $60 \mathrm{mg} /$ day is a safe and effective FDAapproved treatment for DPNP. Duloxetine is cost-saving compared with other approved and non-approved treatments for DPNP.
2. Given its other indications, duloxetine is a particularly good choice for DPNP treatment in patients with coexisting major depressive disorder, generalized anxiety disorder, fibromyalgia, or chronic musculoskeletal pain.

3. Duloxetine use should be avoided in patients with hepatic impairment, severe renal disease, or serious or unstable medical conditions.

4. Routine use of duloxetine doses greater than $60 \mathrm{mg} /$ day for DPNP management are not recommended since higher doses are no more effective, and are associated with more TEAEs and worse tolerability. In patients with an inadequate therapeutic response to duloxetine $60 \mathrm{mg} /$ day, addition of pregabalin or gabapentin is recommended.

5. TEAEs with duloxetine are typical for SNRI medications and include nausea, dizziness, somnolence, fatigue, sweating, dry mouth, constipation, and diarrhea. Nausea, the most common reason for discontinuation of duloxetine in clinical trials, can be limited by taking duloxetine with food.

6. Duloxetine treatment did not increase the risk of $\mathrm{CV}$ events in diabetic patients. However, CV risk factors should be regularly monitored and minimized in all diabetics.

7. No clinically significant mean changes in measures of glycemic control were seen in duloxetine-treated patients. However, since changes in individual patients cannot be predicted, regular monitoring of blood glucose and $\mathrm{HbA}_{1 \mathrm{c}}$ levels are recommended in diabetic patients treated with duloxetine.

8. To maximize tolerability, we recommend starting duloxetine at $20 \mathrm{mg} /$ day with the evening meal or a bedtime snack and increasing in 20-mg increments as needed and tolerated in weekly or bi-weekly increments to a maximum dose of $60 \mathrm{mg} /$ day.

\section{Disclosure}

The authors received no financial or other support from the pharmaceutical industry for the preparation of this manuscript. Drs Ormseth and Sholz have no disclosures. Dr Boomershine has served as a promotional speaker for Eli Lilly and Company and Pfizer Inc, worked as a consultant for Pfizer Inc, and received research funding from Pfizer Inc.

\section{References}

1. Centers for Disease Control and Prevention. National Diabetes Fact Sheet, 2007. Available at: https://apps.nccd.cdc.gov/DDTSTRS/ FactSheet.aspx. Accessed May 20, 2011.

2. Centers for Disease Control and Prevention. Diabetes: disabling, deadly, and on the rise, 2005. Available at: http://www.cdc.gov/diabetes. Accessed May 20, 2011. 
3. National Institute of Diabetes and Digestive and Kidney Diseases. Diabetic Neuropathies: The Nerve Damage of Diabetes. NIH Publication No. 02-3185. May 2002. Available at: http://permanent.access. gpo.gov/lps19677/www.niddk.nih.gov/health/diabetes/pubs/neuro/ neuro.htm. Accessed May 20, 2011.

4. Dyck PJ, Kratz KM, Karnes JL, et al. The prevalence by staged severity of various types of diabetic neuropathy, retinopathy and nephropathy in a population-based cohort: the Rochester Diabetic Neuropathy Study. Neurology. 1993;43:817-824.

5. Treede RD, Jensen TS, Campbell JN, et al. Neuropathic pain: redefinition and a grading system for clinical and research diagnostic purposes. Neurology. 2008;70:1630-1635.

6. Davies M, Brophy S, Williams R, Taylor A. The prevalence, severity, and impact of painful diabetic peripheral neuropathy in type 2 diabetes. Diabetes Care. 2006;29:1518-1522.

7. Galer BS, Gianans A, Jensen MP. Painful diabetic polyneuropathy: epidemiology, pain description, and quality of life. Diabetes Res Clin Pract. 2000;47:123-128.

8. Jensen PG, Larson JR. Management of painful diabetic neuropathy. Drugs Aging. 2001;18:737-749.

9. Veves A, Manes C, Murray HJ, Young MJ, Boulton AJ. Painful neuropathy and foot ulceration in diabetic patients. Diabetes Care 1993;16:1187-1189.

10. Gordois A, Scuffham P, Shearer A, Oglesby A, Tobian JA. The health care costs of diabetic peripheral neuropathy in the US. Diabetes Care. 2003;26:1790-1795.

11. Wu N, Chen S, Boulanger L, et al. Duloxetine compliance and its association with healthcare costs among patients with diabetic periphera neuropathic pain. J Med Economics. 2009;12:192-202.

12. Boule NG, Haddad E, Kenny GP, Wells GA, Sigal RJ. Effects of exercise on glycemic control and body mass in type 2 diabetes mellitus. A metaanalysis of controlled clinical trials. JAMA. 2001;286:1218-1227.

13. American Diabetes Association. Standards of Medical Care in Diabets2010. Diabetes Care. 2010;33(Suppl 1):S11-S61.

14. Quattrini C, Tesfaye S. Understanding the impact of painful diabetic neuropathy. Diabetes Metab Res Rev. 2003;19:S2-S8.

15. Spiegel K, Leproult R, Van Cauter E. Impact of sleep dept on metabolic and endocrine function. Lancet. 1999;354:1435-1439.

16. Pfizer LYRICA DPN Physician ATU Study. February 26, 2007. Pfizer Inc, New York.

17. Berger A, Dukes EM, Oster G. Clinical characteristics and economic costs of patients with painful neuropathic disorders. J Pain. 2004;5:143-149.

18. Sorensen L, Molyneaux L, Yue D. Insensate versus painful diabetic neuropathy: the effects of height, gender, ethnicity and glycemic control Diabetes Res Clin Pract. 2002;57:45-51.

19. Tesfaye S, Selvarajah D. The Eurodiab study: what has this taught us about diabetic peripheral neuropathy? Current Diabetes Reports 2009;9:432-434

20. Gemignani F. Acute painful diabetic neuropathy induced by strict glycemic control ("insulin neuritis"): the old enigma is still unsolved. Biomed Pharmacother. 2009;63:249-250.

21. Gibbons CH, Freeman R. Treatment-induced diabetic neuropathy: a reversible painful autonomic neuropathy. Ann Neurol. 2010;67: 534-541.

22. [No authors listed]. The effect of intensive diabetes therapy on the development and progression of neuropathy. The Diabetes Control and Complications Trial Research Group. Ann Intern Med. 1995;122:561-568.

23. Amthor KF, Dahl-Jorgensen K, Berg TJ, et al. The effect of 8 years of strict glycaemic control on peripheral nerve function in IDDM patients: the Oslo Study. Diabetologia. 1994;37:579-584.

24. Oyibo SO, Prasad YD, Jackson NJ, et al. The relationship between blood glucose excursions and painful diabetic peripheral neuropathy: a pilot study. Diabet Med. 2002;19:870-873.

25. Boulton AJ, Drury J, Clarke B, Ward JD. Continuous subcutaneous insulin infusion in the management of painful diabetic neuropathy. Diabetes Care. 1982;5:386-390.
26. Archer AG, Watkins PJ, Thomas PK, et al. The natural history of acute painful neuropathy in diabetes mellitus. J Neurol Neurosurg Psychiatry. 1983;46:491-499.

27. Cymbalta [package insert]. Indianapolis, IN: Eli Lilly and Company; May 2011.

28. Fields HL, Basbaum AI, Heinricher MM. Central nervous system mechanisms of pain modulation. In: McMahon SB, Koltzenberg M, editors. Wall and Melzack's Textbook of Pain. 5th ed. London, UK: Elsevier, Churchill, Livingston; 2006:125-142.

29. Lyrica [package insert]. New York: Pfizer Inc; 2011 May.

30. ArgoffCE, Backonja MM, Belgrade MJ, et al. Consensus guidelines: treatment planning and options. Mayo Clin Proc. 2006;81(Suppl 4):S12-S25.

31. Bril V, England J, Franklin GM, et al. Evidence-based Guideline: treatment of Painful Diabetic Neuropathy, Report of the American Academy of Neurology, the American Association of Neuromuscular and Electrodiagnostic Medicine, and the American Academy of Physical Medicine and Rehabilitation. Published concurrently in: Neurology. 2011;76:1758-65; Phys Med Rehab. 2011;3:345-352. e21; Muscle Nerve. 2011;43:910-917.

32. Simpson DA. Gabapentin and venlafaxine for the treatment of painful diabetic neuropathy. J Clin Neuromuscul Dis. 2001;3:53-62.

33. Raskin J, Pritchett YL, Wang F, et al. A double-blind, randomized multicenter trial comparing duloxetine with placebo in the management of diabetic peripheral neuropathic pain. Pain Medicine. 2005;6:346-356.

34. Goldstein DJ, Lu Y, Detke M, et al. Duloxetine vs placebo in patients with painful diabetic neuropathy. Pain. 2005;116:109-118.

35. Wernicke JF, Pritchett YL, D'Souza DN, et al. A randomized controlled trial of duloxetine in diabetic peripheral neuropathic pain. Neurology. 2006;67:1411-1420.

36. Lunetta M, Le Moli R, Grasso G, Sangiorgio L. A simplified diagnostic test for ambulatory screening of peripheral diabetic neuropathy. Diabetes Res Clin Practice. 1998;39:165-172.

37. Cleeland CS, Ryan KM. Pain assessment: global use of the Brief Pain Inventory. Ann Acad Med Singapore. 1994;23:129-138.

38. Melzack R. The short-form McGill Pain Questionaire. Pain. 1987;30:191-197.

39. Ashkenazi A, Young WB. Dynamic mechanical (brush) allodynia in cluster headache. Headache. 2004;44:1010-1012.

40. Hamilton M. A rating scale for depression 1960. J Neurol Neurosurg Psychiatry. 1960;23:56-62.

41. Guy W. ECDEI Assessment Manual for Psychopharmacology, Revised. US Department of Health, Education, and Welfare Publication (ADM) Rockville, MD: National Institute of Mental Health; 1976:76-338.

42. Samuelsson M, Leffler A, Johansson B, Hansson P. On the repeatability of brush-evoked allydynia using a novel semi-quantitative method in patients with peripheral neuropathic pain. Pain. 2007;130:40-46.

43. Ware JE, Snow KK, Kosinski M, Gandek B. SF-36 health survey manual and interpretation guide. Boston, MA: The Health Institute, New England Medical Center 1993

44. The EuroQol Group. EuroQol-a new facility for the measurement of health-related quality of life. Health Policy. 1990;16:199-208.

45. Beck AT, Steer RA, Ball R, Ranieri W. Comparison of Beck Depression Inventories -IA and -II in psychiatric outpatients. J Pers Assess. 1996;67:588-597.

46. Beck AT, Epstein N, Brown G, Steer RA. An inventory for measuring clinical anxiety: psychometric properties. J Consult Clin Psychol. 1988;56:893-897.

47. Dworkin RH, Turk DC, Wyrwich KW, et al. Interpreting the clinical importance of treatment outcomes in chronic pain clinical trials: IMMPACT recommendations. J Pain. 2008;9:105-121.

48. Raskin J, Smith TR, Wong K, et al. Duloxetine versus routine care in the long-term management of diabetic peripheral neuropathic pain. $J$ Palliat Med. 2006;9:29-40.

49. Wernicke J, Raskin J, Rosen A, et al. Duloxetine in the long-term management of diabetic peripheral neuropathic pain: an open-label, 52-week extension of a randomized controlled clinical trial. Curr Ther Res Clin Exp. 2006;67:283-304. 
50. Wernicke JF, Wang F, Pritchett YL, et al. An open-label 52-week clinical extension comparing duloxetine with routine care in patients with diabetic peripheral neuropathic pain. Pain Med. 2007;8:503-513.

51. Skljarevski V, Desaiah D, Zhang Q, et al. Evaluating the maintenance of effect of duloxetine in patients with diabetic peripheral neuropathic pain. Diabetes Metab Res Rev. 2009;25:623-631.

52. Raskin J, Wang F, Pritchett YL, Goldstein DJ. Duloxetine for patients with diabetic peripheral neuropathic pain: a 6-month open-label safety study. Pain Med. 2006; 7:373-385.

53. Lunn MP, Hughes RA, Wiffen PJ. Duloxetine for treating painful neuropathy or chronic pain. Cochrane Database Syst Rev. 2009;7: CD007115.

54. Garcia MJ, McNamara PM, Kannel WB. Morbidity and mortality in diabetics in the Framingham population: sixteen-year follow-up study. Diabetes. 1974;23:105-111.

55. American Diabetes Association, the National Heart, Lung and Blood Institute; the Juvenile Diabetes Foundation international; the national Institute of Diabetes and Digestive and Kidney Disease, and the American heart Association. Diabetes mellitus: a major risk factor for cardiovascular disease. Circulation. 1999;100:1132-1133.

56. Sowers JR, Epstein M, Frohlich ED. Diabetes, hypertension, and cardiovascular disease: An update. Hypertension. 2001;37:1053-1059.

57. Maahs DM, Kinney GL, Wadwa P, et al. Hypertension prevalence, awareness, treatment and control in an adult type 1 diabetes population and a comparable general population. Diabetes Care. 2005;28:301-306.

58. Moore WV, Fredrickson D, Brenner A, et al. Prevalence of hypertension in patient with type II diabetes in referral versus primary care clinics. J Diabetes Complications. 1998;12:302-306.

59. Thase ME, Tran PV, Wiltse C, et al. Cardiovascular profile of duloxetine, a dual reuptake inhibitor of serotonin and norepinephrine. J Clin Psychopharmacology. 2005;25:132-140.

60. Wernicke JF, Prakash A, Kajdasz DK, Houston J. Safety and tolerability of duloxetine treatment of diabetic peripheral neuropathic pain between patients with and without cardiovascular conditions. J Diabetes Complications. 2009;23:349-359.

61. McIntyre RS, Soczynska JK, Konarski JZ, Kennedy SH. The effect of antidepressants on glucose homeostasis and insulin sensitivity: synthesis and mechanisms. Expert Opin Drug Saf. 2006;5:157-168.

62. Hardy T, Sachson R, Shen S, et al. Does treatment with duloxetine for neuropathic pain impact glycemic control? Diabetes Care. 2007;30: 21-26.
63. Ziere G, Dieleman JP, van der Cammen TJ, et al. Selective serotonin reuptake inhibiting antidepressants are associated with an increased risk of nonvertebral fractures. J Clin Psychopharmacol. 2008;28:411-417.

64. Raskin J, Smith TR, Wong K, et al. Duloxetine versus routine care in the long-term management of diabetic peripheral neuropathic pain. J Palliat Med. 2006;9:29-40.

65. Christensen JO, Swendsen OL. Bone mineral in pre- and postmenopausal women with insulin dependent and non-insulin dependent diabetes mellitus. Osteoporosis Int. 1999;10:307-311.

66. Stone M, Laughren T, Jones ML, et al. Risk of suicidality in clinical trials of antidepressants in adults: analysis of proprietary data submitted to US Food and Drug Administration. BMJ. 2009;339:b2880.

67. O'Connor AB, Noyes K, Holloway RG. A cost-utility comparison of four first-line medications in painful diabetic neuropathy. Pharmacoeconomics. 2008;26:1045-1064.

68. Wu EQ, Birnbaum HG, Mareva MN, et al. Cost-effectiveness of duloxetine versus routine treatment for US patients with diabetic peripheral neuropathic pain J Pain. 2006;7:399-407.

69. Quilici S, Chancellor J, Lothgren M, et al. Meta-analysis of duloxetine vs pregabalin and gabapentin in the treatment of diabetic peripheral neuropathic pain. BMC Neurology. 2009;9:6.

70. Chen SY, Wu N, Boulanger L, et al. The relationship between average daily dose, medication adherence, and health-care costs among diabetic peripheral neuropathic pain patients initiated on duloxetine therapy. Pain Pract. 2010;10:530-539.

71. Zhao Y, Wu N, Chen S, et al. Changes in opioid use and healthcare costs among US patients with diabetic peripheral neuropathic pain treated with duloxetine compared with other therapies. Curr Med Res Opin. 2010;26:2147-2156.

72. Solomon DH, Rassen JA, Glynn RF, et al. The comparative safety of analgesics in older adults with arthritis. Arch Intern Med. 2010;170: 1968-1976.

73. Shelton RC. The nature of the discontinuation syndrome associated with antidepressant drugs. J Clin Psychiatry. 2006;67(Suppl 4):3-7.

74. Perahia DG, Kajdasz DK, Desaiah D, Haddad PM. Symptoms following abrupt discontinuation of duloxetine treatment in patients with major depressive disorder. J Affect Disord. 2005;89:207-212.

75. Perahia DG, Quail D, Desaiah D, et al. Switching to duloxetine from selective serotonin reuptake inhibitor antidepressants: a multicenter trial comparing 2 switching techniques. J Clin Psychiatry. 2008;69: 95-105.
Patient Preference and Adherence

\section{Publish your work in this journal}

Patient Preference and Adherence is an international, peer-reviewed, open access journal focusing on the growing importance of patient preference and adherence throughout the therapeutic continuum. Patient satisfaction, acceptability, quality of life, compliance, persistence and their role in developing new therapeutic modalities and compounds to

\section{Dovepress}

optimize clinical outcomes for existing disease states are major areas of interest. This journal has been accepted for indexing on PubMed Central. The manuscript management system is completely online and includes a very quick and fair peer-review system. Visit http://www.dovepress.com/ testimonials.php to read real quotes from published authors. 